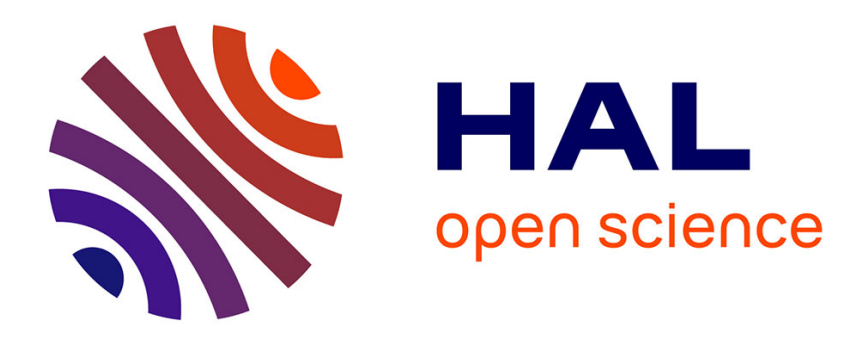

\title{
Étude d'un réacteur plasmique bicolonne et utilisation en source de réactifs chimiques
}

\author{
M. Fabry, A. Vicherat, M. Felden
}

\section{To cite this version:}

M. Fabry, A. Vicherat, M. Felden. Étude d'un réacteur plasmique bicolonne et utilisation en source de réactifs chimiques. Revue de Physique Appliquée, 1976, 11 (5), pp.581-585. 10.1051/rphysap:01976001105058100 . jpa-00244092

\section{HAL Id: jpa-00244092 https://hal.science/jpa-00244092}

Submitted on 1 Jan 1976

HAL is a multi-disciplinary open access archive for the deposit and dissemination of scientific research documents, whether they are published or not. The documents may come from teaching and research institutions in France or abroad, or from public or private research centers.
L'archive ouverte pluridisciplinaire HAL, est destinée au dépôt et à la diffusion de documents scientifiques de niveau recherche, publiés ou non, émanant des établissements d'enseignement et de recherche français ou étrangers, des laboratoires publics ou privés. 
Classification

Physics Abstracts

6.580

\title{
ÉTUDE D'UN RÉACTEUR PLASMIQUE BICOLONNE ET UTILISATION EN SOURCE DE RÉACTIFS CHIMIQUES
}

\author{
M. FABRY, A. VICHERAT et M. FELDEN \\ Laboratoire de Physique des Milieux Ionisés de l'Université de Nancy I \\ C. O. 140. 54037 Nancy Cedex, France
}

(Reçu le $1^{\text {er }}$ mars 1976, accepté le 14 avril 1976)

\begin{abstract}
Résumé. - Nous avons conçu et réalisé un générateur double colonne adapté à la production d'espèces excitées. Nous étudions tout d'abord le fonctionnement monocolonne pour tester différentes techniques de diagnostic, puis nous utilisons le générateur bicolonne afin d'étudier les mélanges de gaz moléculaires et les possibilités de synthèse de composés plus complexes.

Abstract. - We report the conception and realization of a double column generator adapted for the production of excited species. Following a study of the operation of a single plasma column in order to test different diagnostic techniques we describe the use of a double column for studying the mixing of molecular gases and the possibility of synthesis of more complex compounds.
\end{abstract}

1. Introduction. - L'utilisation des plasmas dans le cadre de la chimie concerne en particulier les applications industrielles des décharges électriques dans les gaz. Ces études, qui deviennent très vite complexes sur le plan théorique par la nature du milieu puisque les divers composants sont hors d'équilibre, évoluent actuellement dans trois directions différentes. La première comprend le domaine des recherches dans l'ionosphère, rendu nécessaire par les progrès de l'astronautique, des télécommunications, de la météorologie $[1,2,3]$. La deuxième comprend une branche de la chimie des décharges qui s'intéresse essentiellement aux relations vibration-vibration de molécules excitées par une décharge électrique [4]. Les systèmes les plus étudiés sont les lasers à mélange ternaire $\mathrm{CO}_{2}-\mathrm{N}_{2}-\mathrm{He}$ ou d'autres systèmes comportant des molécules plus compliquées comme le benzène. Enfin, le plus vaste champ d'étude reste l'utilisation des décharges à des fins de synthèse $[5,6]$. La grande difficulté réside, en dehors du traitement théorique, dans le manque de sélectivité des réactions chimiques de plasmas, d'où un problème important de trempe et de piégeage des produits. Un autre facteur limitant l'utilisation industrielle, est dû à la faible pression existant dans le réacteur ce qui conduit à des rendements quantitatifs faibles.

Nous avons envisagé la réalisation d'un générateur reflex comportant deux colonnes de plasma, le cas échéant de nature différente adapté à la production d'espèces excitées neutres ou ionisées et permettant l'étude des réactions entre les deux milieux ionisés qui sont confinés dans la même enceinte.
2. Technologie du réacteur plasmochimique bicolonne. - Le générateur se compose essentiellement d'une enceinte à vide cylindrique d'une longueur de $116 \mathrm{~cm}$ et d'un diamètre de $9,4 \mathrm{~cm}$. Le pompage, devant répondre aux impératifs suivants : ne pas polluer, avoir un rendement efficace entre $10^{-2}$ et $10^{-7}$ torr et ne pas être sélectif, est effectué par une pompe turbomoléculaire. A chaque extrémité de l'enceinte, nous disposons une platine porte-électrodes comportant à la fois l'injection de gaz et le refroidissement des cathodes. Celles-ci sont des disques de $30 \mathrm{~mm}$ de diamètre en duralumin, choisi pour son pouvoir d'émission secondaire à basse énergie et parce que très bon conducteur de la chaleur. Les anodes posent moins de problèmes ; elles ont été réalisées en molybdène avec un diamètre légèrement supérieur à celui des cathodes $(35 \mathrm{~mm})$ de manière à réduire la diffusion et obtenir une colonne de plasma assez importante. Le refroidissement est suffisant sauf à basse pression $\left(\sim 10^{-4}\right.$ torr $)$ où les températures électroniques élevées ont pour conséquence de porter au rouge l'anode ce qui peut provoquer une légère métallisation des surfaces environnantes et en particulier des isolants. Une vue d'ensemble d'une extrémité du générateur avec les deux jeux d'électrodes est représentée sur la figure 1. L'espace que nous avons cherché à rendre minimal entre les deux colonnes de plasma a nécessité une isolation électrique des deux systèmes d'électrodes par des tubes en verre que nous avons prolongés jusqu'au voisinage du centre de la machine pour limiter les interférences entre les deux décharges et ménager un espace réactionnel au centre de l'enceinte. L'injection de gaz se fait à travers les 


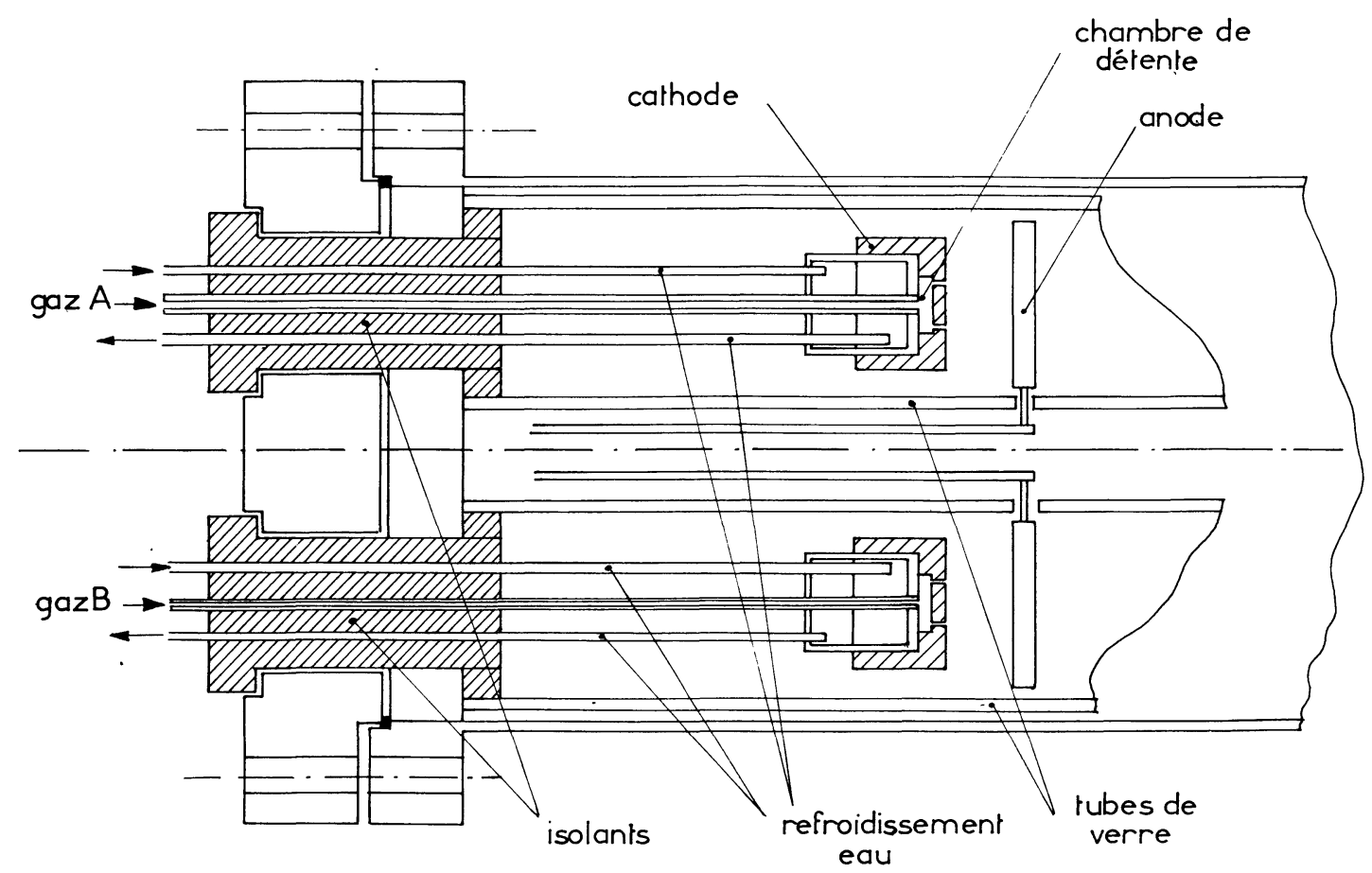

FIG. 1. - Ensemble anode-cathode avec les injections de gaz et les systèmes de refroidissement.

cathodes par 40 trous de $0,3 \mathrm{~mm}$ situés sur le pourtour d'une chambre de détente (Fig. 1). Les deux jeux d'électrodes ont été disposés dans un plan vertical afin de permettre l'observation des deux colonnes à travers les fenêtres de l'enceinte. Le système fonctionne avec satisfaction jusqu'à des courants pouvant atteindre $1 \mathrm{~A}$ sur des faibles durées.

Le champ magnétique, créé par six bobines refroidies, peut atteindre $3000 \mathrm{G}$ avec des ondulations inférieures ou égales à $10 \%$ du champ maximum. Le champ électrique est fourni pour chaque système d'électrodes par deux alimentations indépendantes, d'une puissance de $12 \mathrm{~kW}$ et régulées en courant. Nous $\mathrm{y}$ avons adjoint des alimentations de faible puissance destinées à polariser une colonne par rapport à l'autre. Le contrôle de la pression se fait à l'aide de trois jauges : une Pirani, une Penning et une jauge à ionisation à filament couplée à une vanne d'injection automatique du gaz assurant une très grande stabilité de la pression en cours de fonctionnement.

3. Etude du fonctionnement du générateur. Résultats expérimentaux. - 3.1 FonCTIONNEMENT MONOCOLONNE. - L'étude du fonctionnement du générateur commence par la détermination des caractéristiques en monocolonne. La caractéristique courant-tension de la décharge s'apparente de très près à une caractéristique d'arc avec trois zones. Elle varie essentiellement selon la nature du gaz. L'étude de l'influence du champ magnétique permet de déterminer deux modes de fonctionnement principaux que l'on pourra qualifier de basse et haute induction. Pour un champ magnétique faible $(B=700 \mathrm{G})$ nous obtenons la variation de la tension de décharge $V_{\mathrm{d}}$ en fonction de la pression $P$

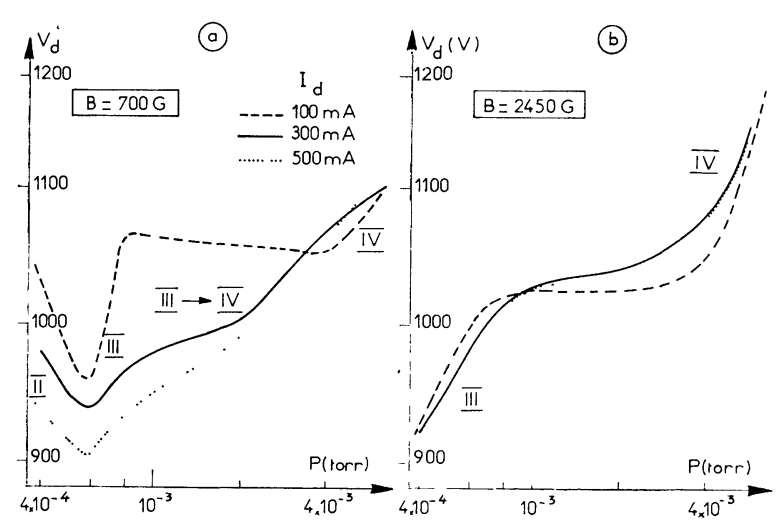

Fig. 2. - Modes de fonctionnement de la décharge monocolonne pour différents champs magnétiques $B$ et courants de décharge $I_{\mathrm{d}}$.

indiquée sur la figure $2 a$. Nous constatons la présence de différents régimes de fonctionnement apparentés aux modes II-III et IV décrits par Hooper [7], la transition III $\rightarrow$ IV étant marquée pour les faibles courants de décharge. Par contre, pour un champ magnétique plus élevé ( $B=2450 \mathrm{G})$ nous obtenons la variation présentée sur la figure $2 b$, c'est-à-dire qu'un glissement des modes vers les basses pressions s'effectue au fur et à mesure que le champ magnétique augmente et le mode II ne peut plus être obtenu. Cette étude sommaire des modes de fonctionnement de la décharge sera à rapprocher des résultats obtenus pour les mesures de la température vibrationnelle pour différentes conditions expérimentales.

3.2 FonCTIONNEMENT BICOLONNE. - Nous étudions dans ce paragraphe le fonctionnement en double 
colonne afin de connaître l'influence électrique d'une colonne sur l'autre. Il est en effet important de pouvoir faire varier les caractéristiques électriques d'une colonne sans que les paramètres de l'autre colonne en soient affectés. Après nous être assurés qu'aucune interconnexion électrique ne pouvait se faire entre les alimentations ou les circuits de refroidissement (nécessairement séparés), nous nous sommes intéressés à l'étude des caractéristiques de fonctionnement, simultanément sur les deux colonnes. Si nous fixons un point de fonctionnement sur une colonne A et que nous faisons varier les caractéristiques courant-tension sur la seconde colonne $\mathrm{B}$, nous constatons que ces variations n'induisent aucune modification sur le fonctionnement de la colonne A si le courant de décharge de celle-ci est supérieur à $150 \mathrm{~mA}$. Par contre, pour un courant plus faible correspondant à un fonctionnement proche de l'amorçage, nous constatons une influence électrique d'une colonne sur l'autre. En particulier, la présence d'une décharge influe sur l'amorçage de la seconde décharge, celui-ci étant favorisé par la présence de charges primaires. Ainsi, si nous travaillons à courant suffisamment élevé, les deux colonnes sont électriquement indépendantes.

En ce qui concerne l'indépendance gazeuse, elle est réalisée au niveau des cathodes grâce à la présence des tubes en verre limitant la diffusion (Fig. 1) mais le centre de la décharge est constitué par un mélange des deux gaz, d'où la possibilité de réactions dans cette partie.

3.3 Méthodes de diagnostic. - Pour analyser les espèces présentes dans le milieu réactionnel, trois techniques principales ont été utilisées. La première est la spectroscopie optique d'émission visible et proche UV qui permet, d'une part, la mesure de la température vibrationnelle $T_{\mathrm{v}}$ et de la température rotationnelle $T_{\mathrm{r}}$ et, d'autre part, l'identification de composés chimiques par l'étude de leurs bandes moléculaires. La spectrométrie de masse avec extraction latérale, constitue la deuxième méthode, elle permet d'identifier les ions et les neutres présents dans la décharge et complète le diagnostic optique. Enfin, la troisième méthode est une technique de sonde spécifique aux décharges en champs magnétique, permettant de mesurer la température ionique $T_{\mathrm{i}}$ : les sondes sensibles aux ions (ISP) utilisant la différence des rayons de Larmor des ions et des électrons. La température électronique est mesurée à partir des sondes de Langmuir habituelles.

Reprenons ces différentes méthodes de diagnostic et indiquons les résultats obtenus dans une décharge d'azote. Nous avons choisi ce gaz pour effectuer une étude complète par voie optique, les différentes données numériques nécessaires à cette étude étant connues et suffisamment précises tant pour $\mathrm{N}_{2}$ que pour $\mathrm{N}_{2}^{+}$.

3.3.1 Spectroscopie d'émission. - Le montage optique utilisé est essentiellement constitué de hublots et lentilles en quartz et d'un monochromateur à réseau.
Si nous nous intéressons à une transition vibrationnelle, l'intensité de la bande s'écrit :

$$
I_{\mathrm{v}^{\prime} \mathrm{v}^{\prime \prime}}=\frac{64 \pi^{4} c}{3} N_{\mathrm{v}^{\prime}} \sigma_{\mathrm{v}^{\prime} \mathrm{v}^{\prime \prime}}^{4} S_{\mathrm{v}^{\prime} \mathrm{v}^{\prime \prime}}
$$

où $N_{\mathrm{v}^{\prime}}$ est la population de niveau vibrationnel $\mathrm{v}^{\prime}$ de l'état électronique supérieur, $\sigma_{\mathrm{v}^{\prime} \mathrm{v}^{\prime \prime}}$ le nombre d'ondes de la transition $\mathrm{v}^{\prime} \rightarrow \mathrm{v}^{\prime \prime}$ et $S_{\mathrm{v}^{\prime} \mathrm{v}^{\prime \prime}}$ un facteur qui dépend de la transition considérée, de deux états électroniques inférieur et supérieur ainsi que de $\mathrm{v}^{\prime}$ et $\mathrm{v}^{\prime \prime}$. Pour l'azote, nous utilisons les valeurs données par Nicholls [8] pour le système second positif de $\mathrm{N}_{2}\left(C^{3} \pi_{\mathrm{u}} \rightarrow \mathrm{B}^{3} \pi_{\mathrm{g}}\right)$ et pour le système premier négatif de $\mathrm{N}_{2}^{+}\left(\mathrm{B}^{2} \Sigma_{\mathrm{u}}^{+} \rightarrow \mathrm{X}^{2} \Sigma_{\mathrm{j}}^{+}\right)$. En faisant l'hypothèse d'une distribution de Boltzmann sur les niveaux vibrationnels :

$$
N_{\mathrm{v}^{\prime}}=N_{0} \exp \left(-h c G_{\mathrm{v}^{\prime}} / k T_{\mathrm{v}}\right)
$$

nous pourrons déduire la température $T_{\mathrm{v}}$ de la pente de la droite obtenue en portant

$$
\ln \left[I_{\mathrm{v}^{\prime} \mathrm{v}^{\prime \prime}} / T_{\mathrm{v}^{\prime} \mathrm{v}^{\prime \prime}}^{4} S_{\mathrm{v}^{\prime} \mathrm{v}^{\prime \prime}}\right]
$$

en fonction de $G_{\mathrm{v}^{\prime}}$. Nous avons tout d'abord vérifié l'hypothèse de la distribution de Boltzmann pour la population de niveaux vibrationnels, puis étudié la variation de la température vibrationnelle $T_{\mathrm{v}}$ en fonction de la pression, pour différents courants de décharge (Fig. 3). Pour les neutres (Fig. 3a), nous constatons une très faible dépendance de $T_{\mathrm{v}}$ vis-à-vis de la pression. Par

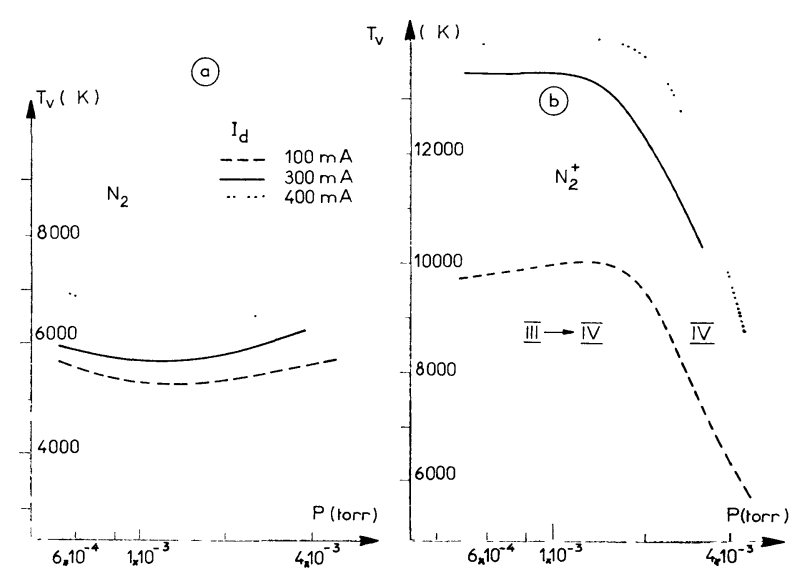

FIG. 3. - Variation de la température vibrationnelle $T_{\mathrm{v}}$ en fonction de la pression.

contre, pour les ions (Fig. $3 b$ ) nous obtenons une température vibrationnelle nettement plus élevée et dépendant fortement de la pression. Si nous comparons cette figure à la figure 2 , nous pouvons rapprocher les variations de $T_{\mathrm{v}}$ avec les changements de mode de fonctionnement de la décharge réflex. En particulier, dans le mode IV nous constatons une chute brutale de $T_{\mathrm{v}}$ quand la pression augmente. La variation de $T_{\mathrm{v}}$ avec le courant de décharge est similaire sur les courbes $a$ et $b$ et se traduit par une augmentation de $T_{\mathrm{v}}$ avec le courant. En ce qui concerne la température rotationnelle $T_{\mathrm{r}^{\prime}}$ mesurée à partir de la bande $(0,1)$ du système premier 
négatif de $\mathrm{N}_{2}^{+}$, nous obtenons des valeurs nettement plus basses $(\sim 1000 \mathrm{~K})$ et voisines de la température du gaz.

3.3.2 Spectrométrie de masse. - Les avantages de cette technique de diagnostic sont dus à sa sensibilité, à sa grande souplesse d'emploi et à la possibilité d'une grande vitesse de balayage permettant l'observation de phénomènes transitoires. Nous avons choisi un filtre quadrupolaire équipé d'un multiplicateur d'électrons, notre but étant essentiellement de tester qualitativement le milieu à étudier. Il est situé transversalement entre les deux colonnes, dans la zone de diffusion libre. L'efficacité du blindage magnétique a été vérifiée : jusque $1700 \mathrm{G}$, la réponse n'est pas affectée alors qu'au-delà, nous constatons une légère diminution de l'amplitude des différents pics, due à une défocalisation du pinceau d'ions après la source d'ionisation. L'étude du spectre de gaz résiduels a montré la nécessité d'un étuvage à $150^{\circ} \mathrm{C}$ de l'enceinte se traduisant par une diminution de l'amplitude des pics dans un rapport voisin de 100 . Cette méthode de diagnostic sera principalement utilisée pour l'étude du mélange de gaz (§ 4).

3.3.3 Sondes ISP. - L'utilisation des sondes électrostatiques pour la mesure des paramètres de plasma pose des problèmes délicats en champ magnétique. La seule mesure que nous puissions faire est la température électronique $T_{\mathrm{e}}$ à partir des caractéristiques de sondes électrostatiques ordinaires. La mesure des paramètres ioniques n'est plus possible en présence d'un champ magnétique car celui-ci perturbe l'espace grille-collecteur d'un analyseur à grille. Toutefois, de la différence entre les rayons de Larmor des ions et des électrons, vient la possibilité de séparer ceux-ci par sonde ISP [9, 10] : la sonde collecte tous les électrons sur une garde et la mesure du courant ionique peut être effectuée sur une autre électrode. La figure 4 montre la réalisation pratique d'une telle sonde à sélection radiale et une caractéristique ionique type. Nous mesurons une température ionique $T_{\mathrm{i}} \sim 6000 \mathrm{~K}$ alors que la température électronique $T_{\mathrm{e}}$ mesurée à l'aide d'une sonde de Langmuir habituelle est voisine de 80000 à $90000 \mathrm{~K}$.

Les différents résultats obtenus par ces méthodes de diagnostic et récapitulés dans le tableau I montrent le caractère très hors d'équilibre de la décharge mais indiquent qu'un tel générateur produit des espèces excitées neutres et ionisées et peut être utilisée comme source de réactifs chimiques.
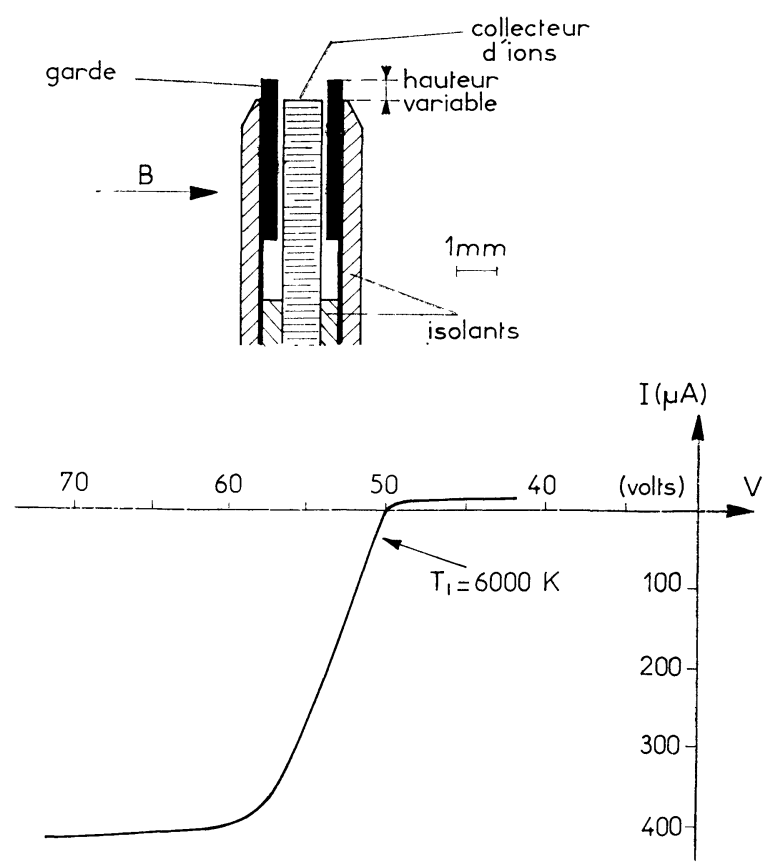

Fig. 4. - Sonde à sélection radiale sensible aux ions et caractéristique $I(V)$ obtenue.

4. Mélanges binaires de gaz moléculaires. - Dans cette partie, nous injectons des gaz différents dans chaque colonne et nous étudions les possibilités de formation de molécules plus complexes. Les premières expériences, effectuées sur un mélange $\mathrm{CO}_{2}-\mathrm{N}_{2}$ montrent que les deux colonnes présentent une indépendance gazeuse au niveau des cathodes alors que le centre de la décharge est constitué par un mélange des deux gaz. Cette étude a été effectuée en mesurant la variation des intensités des raies émises le long d'une colonne. Nous avons ainsi la possibilité de modifier séparément les paramètres de la décharge au niveau de chaque ensemble d'électrodes et d'étudier le mélange des gaz au centre de l'enceinte.

4.1 MÉlANGE $\mathrm{CO}_{2}-\mathrm{N}_{2}$. - En travaillant à pression totale et champ magnétique constants, nous avons relevé les caractéristiques électriques en fonction de la composition du mélange (Fig. 5a). Nous constatons pour les forts courants un maximum pour une composition de $60 \% \mathrm{CO}_{2}-40 \% \mathrm{~N}_{2}$. Dans cette zone correspondant au mode de transition vers les fonctionnements en haute pression, la tension ne dépend que de la composition du gaz et du courant. Ainsi, la présence

\section{TABLEAU I}

\section{Différents paramètres mesurés dans une décharge d'azote et technique de diagnostic utilisée}

Température électronique

Sonde de Langmuir

80000 à $100000 \mathrm{~K}$

\author{
Température \\ ionique
}

Sonde ISP

$$
\begin{gathered}
\text { Température } \\
\text { vibrationnelle } \\
\text { (neutres) }
\end{gathered}
$$

Spectroscopie
Température (ions)

Spectroscopie
Température rotationnelle

Spectroscopie 


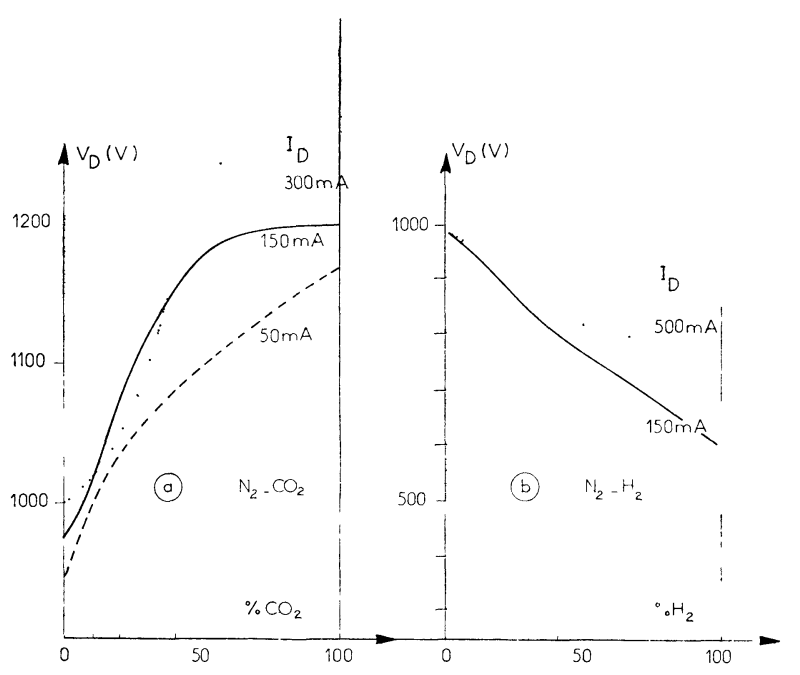

FIG. 5. - Mélanges de gaz moléculaires : a) $\left.\mathrm{N}_{2}-\mathrm{CO}_{2}, b\right) \mathrm{H}_{2}-\mathrm{N}_{2}$. Variation de la tension de décharge en fonction de la composition du mélange $\left(B=1400 \mathrm{G}, p=2 \times 10^{-3}\right.$ torr $)$.

de ce maximum peut-elle s'expliquer par la création de radicaux dont l'énergie de dissociation est supérieure à celle de $\mathrm{N}_{2}$ et $\mathrm{CO}_{2}$. En fait, le spectre optique d'une telle décharge montre la superposition du spectre de $\mathrm{N}_{2}$ et de $\mathrm{CO}_{2}$ pur ainsi qu'une bande caractéristique de $\mathrm{CO}^{+}$(système Comet Tail : $\mathrm{A}^{2} \pi_{\mathrm{i}} \rightarrow \mathrm{X}^{2} \Sigma^{+}[11]$ ). L'étude au spectromètre de masse confirme la forte dissociation de $\mathrm{CO}_{2}$ (mise en évidence de $\mathrm{C}^{+}, \mathrm{O}^{+}$, $\mathrm{CO}^{+}$) et montre l'absence de $\mathrm{CN}^{+}$(dont l'énergie de dissociation est faible) dans la décharge.

4.2 Mélange $\mathrm{N}_{2}-\mathrm{O}_{2}$. - Ce mélange présente une grande analogie avec le mélange $\mathrm{N}_{2}-\mathrm{CO}_{2}$. Il y a apparition d'un maximum très net de la tension pour une composition moyenne de $75 \% \mathrm{~N}_{2}$ et $25 \% \mathrm{O}_{2}$. Comme précédemment, ce maximum tient à la nature du milieu et à la production de NO. Ce fait est confirmé par l'étude au spectromètre de masse montrant la présence de $\mathrm{NO}^{+}$d'autant plus marquée que nous sommes proche de la composition $75 \% \mathrm{~N}_{2}-25 \% \mathrm{O}_{2}$. Le spectre d'émission de $\mathrm{NO}^{+}$se situant à des longueurs d'ondes inférieures à $2000 \AA$ [11], l'étude optique ne nous apporte pas de précisions complémentaires.

Ce mélange présente un intérêt certain pour les études de l'ionosphère ainsi que celles concernant la pollution atmosphérique.

4.3 MÉlanges $\mathrm{H}_{2}-\mathrm{CO}_{2} ; \mathrm{H}_{2}-\mathrm{O}_{2} ; \mathrm{H}_{2}-\mathrm{N}_{2}$. - Nous avons regroupé ces mélanges comportant de l'hydro- gène car ils présentent une grande similitude. Dans les caractéristiques de fonctionnement (Fig. $5 b$ pour $\mathrm{H}_{2}$ $\mathrm{N}_{2}$ ), nous n'obtenons pas de maximum, mais seulement une variation quasi linéaire de la tension quel que soit le courant de décharge. Ainsi, il ne semble pas y avoir création de composés à forte énergie de dissociation. Tout au plus, peut-on noter l'apparition d'un léger minimum dans le mélange $\mathrm{H}_{2}-\mathrm{O}_{2}$ vers $25 \% \mathrm{H}_{2}-75 \%$ $\mathrm{O}_{2}$ correspondant à une possibilité d'ionisation plus efficace sur $\mathrm{OH}$. La spectroscopie optique nous indique la formation de $\mathrm{NH}$ (transition observée $\mathrm{A}^{3} \pi_{\mathrm{i}} \rightarrow \mathrm{X}^{3} \Sigma^{-}$) dans le mélange $\mathrm{N}_{2}-\mathrm{H}_{2}$ et de $\mathrm{OH}$ (transition observée $\mathrm{A}^{2} \Sigma^{+} \rightarrow \mathrm{X}^{2} \pi_{\mathrm{i}}$ ) dans le mélange $\mathrm{O}_{2}-\mathrm{H}_{2}$. Pour le mélange $\mathrm{CO}_{2}-\mathrm{H}_{2}$, ni l'étude optique, ni la spectroscopie de masse ne montrent la formation de composés. Le mélange le plus intéressant semble être $\mathrm{N}_{2}-\mathrm{H}_{2}$ puisque celui-ci nous montre une profusion d'ions extraits et il ne faut pas exclure la synthèse à partir de $\mathrm{NH}$ de molécules plus élaborées telles que $\mathrm{NH}_{2}^{+}, \mathrm{N}_{2} \mathrm{H}^{+}, \mathrm{N}_{2} \mathrm{H}_{2}^{+} \ldots$ L'étude sommaire des mélanges appelle deux remarques : dans la décharge proprement dite, seuls les processus à haute énergie sont réalisés et la décharge est une sorte de réservoir de réactifs, qu'il convient d'utiliser en dehors de la colonne si l'on veut réaliser des synthèses.

5. Conclusion. - Nous avons réalisé un générateur reflex double colonne adapté à la production d'espèces excitées neutres ou ionisées. Nous avons constaté le caractère hors d'équilibre d'une telle décharge et le fort niveau d'excitation et d'ionisation. Elle constitue donc une source de réactifs. Ensuite, l'étude préliminaire des mélanges montre que la synthèse ne peut avoir lieu dans la décharge pour des molécules complexes. Par contre, le générateur permet de fixer et d'étudier les différents paramètres qui influent sur le mode de fonctionnement et la nature du milieu. Un développement vers une utilisation plus chimique implique une pression plus élevée et un générateur dont la géométrie devrait être plus compacte. D'autre part, l'utilisation optimale correspond à une source d'ions et de neutres excités, et non un réacteur, de telle sorte que les radicaux NO, NH, OH... peuvent être utilisés pour des réactions chimiques. Tel qu'il est actuellement conçu, le générateur présente l'intérêt de permettre des études plus axées vers les processus élémentaires (recombinaison, population des niveaux...) rencontrés dans la décharge, ceci grâce à la possibilité d'implantation de moyens de diagnostic divers.

\section{Bibliographie}

[1] Danilov, A. D. et Ivanoy-Kholodnyi, G. S., Sov. Phys. Usp. 8 (1965) 92.

[2] Takayanagi, K. et Itikawa, Y., Space Sci. Rev. 11 (1970) 380.

[3] Ferguson, E. E., Rev. Geophys. Space Phys. 12 (1974) 703.

[4] Dzhidzhoev, M. S., Planonenko, V. T. et Khokhlov, R. V., Sov. Phys. Usp. 13 (1970) 247.

[5] IBERSON, V. J., High Temp. 1 (1969) 243.

[6] Venugopalan, M., Reactions under plasma conditions (John Wiley et Sons, Inc.) 1971.
[7] Hooper Jr.,E. B., A Review of reflex and Penning Discharges in Advances in Electronics and Electron Physics (Marton L. éd.) 1969, p. 295.

[8] Nichools, R. W., Ann. Geophys. 20 (1964) 144.

[9] Nobata, K., Japan J. Appl. Phys. 2 (1963) 719.

[10] Dote, T. et Amenya, H., J. Phys. Soc. Japan 19 (1964) 1915.

[11] Bosen, R., Données spectroscopiques relatives aux molécules diatomiques (Pergamon Press) 1970. 